\title{
Dietary and physical activity behaviours related to obesity-specific quality of life and work productivity: baseline results from a worksite trial
}

\author{
Stephanie Whisnant Cash $^{1}$, Shirley A. A. Beresford ${ }^{1,2 *}$, Jo Ann Henderson ${ }^{2}$, Anne McTiernan ${ }^{1,2}$, \\ Liren $\mathrm{Xiao}^{2}$, C. Y. Wang ${ }^{2}$ and Donald L. Patrick ${ }^{3}$ \\ ${ }^{1}$ Department of Epidemiology, School of Public Health, University of Washington, 1959 NE Pacific Street, Box 357236, \\ Seattle, WA 98195, USA \\ ${ }^{2}$ Fred Hutchinson Cancer Research Center, 1100 Fairview Avenue North, Mailstop M3-B232, Seattle, WA 98109, USA \\ ${ }^{3}$ School of Public Health, University of Washington, Box 359455, 4333 Brooklyn Avenue NE, Room 14-101, Seattle, \\ WA 98195, USA
}

(Submitted 6 June 2011 - Final revision received 14 October 2011 - Accepted 18 October 2011 - First published online 6 December 2011)

\begin{abstract}
Obesity is associated with impaired health-related quality of life (QOL) and reduced productivity; less is known about the effect of dietary factors. The present study investigated how dietary behaviours, physical activity and BMI relate to weight-specific QOL and work productivity. The study was conducted in thirty-one small blue-collar and service industry worksites in Seattle. Participants were 747 employees (33.5\% non-White). Measures included self-reported servings of fruits and vegetables, dietary behaviours such as fast food consumption, Godin free-time physical activity scores, measured height and weight, Obesity and Weight-Loss QOL (OWLQOL) scores, and Work Limitations Questionnaire scores. Baseline data were analysed using linear mixed models separately for men ( $n$ 348) and women ( $n$ 399), since sex modified the effects. BMI was negatively associated with OWLQOL in both women $(P<0 \cdot 001)$ and men $(P<0 \cdot 001)$. The linear effect estimate for OWLQOL scores associated with a one-category increase in BMI was 30 (95\% CI 25 , 44$) \%$ for women and $14(95 \%$ CI 10, 17) \% for men. BMI was positively associated with productivity loss only in women (exp(slope) $=1.46$, $95 \%$ CI $1 \cdot 02,2 \cdot 11, P=0 \cdot 04)$. Eating while doing another activity was negatively associated with OWLQOL scores in men $(P=0 \cdot 0006$, independent of BMI $)$ and with productivity in women $(P=0 \cdot 04$, although the effect diminished when adjusting for BMI). Fast-food meals were associated with decreased productivity in men $(P=0.038$, independent of BMI). The results suggest that obesogenic dietary behaviours and higher BMI are associated with decreased QOL and productivity to different degrees in women and men.
\end{abstract}

Key words: Dietary behaviours: Obesity: Quality of life: Work productivity

Obesity has become a major public health problem. In the USA, the prevalence of obesity (BMI of $30 \mathrm{~kg} / \mathrm{m}^{2}$ or higher) has increased drastically, from $15 \%$ in 1985 to the current $34.3 \%{ }^{(1)}$. It is well established that obesity is associated with a number of adverse health outcomes including hypertension, type 2 diabetes, heart disease, stroke, respiratory problems and certain types of cancer ${ }^{(2)}$. Often overlooked are the equally important relationships between obesity and nonphysical health outcomes, such as well-being, productivity and psychosocial functioning.

To accommodate this oversight, Healthy People $2010^{(3)}$ expanded its 2000 goal of longevity to include increased quality of life (QOL) and overall well-being ${ }^{(4)}$; researchers have started collecting such data, and the body of literature concerning obesity and outcomes such as QOL and productivity is rapidly growing. Health-related QOL (HRQOL) has been defined variously, but most often concerns concepts of physical, psychological, and social functioning and well-being. Measures of perceived QOL are confined to those aspects of life known only to the person and are perceptions about how a specific condition affects a person's life in relation to their goals, standards and concerns ${ }^{(5)}$.

Several studies have found that obesity is associated with a diminished HRQOL ${ }^{(6,7)}$, and higher BMI has been shown to be associated with physical, social and emotional domains of $\mathrm{HRQOL}^{(4)}$. Recently, researchers have also examined obesity-specific subscales of HRQOL. Kolotkin et al. ${ }^{(7)}$, using the Impact of Weight on Quality of Life-Lite instrument, showed that individuals with higher BMI were more likely to report impaired obesity-specific HRQOL. Similar impairments

Abbreviations: HRQOL, health-related quality of life; MOVE 'M, Move and Moderate in Balance; OWLQOL, Obesity and Weight-Loss Quality of Life; WLQ, Work Limitations Questionnaire. 
in energy and vitality, in addition to increased body pain, have been observed in obese patients ${ }^{(6)}$.

Obesity is also associated with reduced productivity ${ }^{(8)}$, with an estimated $\$ 73.1$ billion in productivity losses due to obesity among full-time employees ${ }^{(9)}$. While some of this effect may be due to co-morbid conditions such as diabetes and hypertension, evidence suggests that lower productivity is exacerbated by the presence of obesity ${ }^{(10)}$. BMI appears to independently predict absenteeism, with obese employees missing more days of work than their normal-weight counterparts $^{(11)}$. With high estimated costs lost due to sickness or disability related to obesity, the prevalence of obesity is a serious economic concern.

Evidence suggests that HRQOL and productivity are modifiable by targeting diet and exercise. Randomised controlled trials have shown that a diet rich in fruits and vegetables is associated with higher $\mathrm{HRQOL}^{(12)}$. Data also suggest that physical activity is associated with improved HRQOL ${ }^{(13)}$ and fewer physical and mental unhealthy days ${ }^{(14,15)}$. In addition, data from the longitudinal Swedish Obesity Study, which used the Swedish Obesity Study Quality of Life Survey, showed that weight loss was associated with improved scores on the obesity-related problems subscale ${ }^{(4)}$. Worksite interventions have proven successful in increasing fruit and vegetable consumption and physical activity ${ }^{(16)}$, and may indirectly increase HRQOL and productivity.

The Move and Moderate in Balance (MOVE 'M) study is a worksite-randomised trial to encourage healthy dietary choices and physical activity and maintain or reduce the weight of employees at worksites. The present study explores baseline associations between BMI, fruit and vegetable consumption, physical activity and weight-related QOL and proxy measures for productivity among employees of small South Seattle worksites.

\section{Methods}

\section{Recruitment}

Worksites in South Seattle were recruited from a defined geographical area and were identified using US Standard Industrial Classification two-digit $\operatorname{codes}^{(17)}$, including construction, manufacturing, transportation and service industries. Worksites with between fifteen and eighty employees were recruited. During a screening call, additional criteria were checked: a high proportion of employees with sedentary or stationary occupations $(>50 \%)$, a low turnover rate $(<30 \%)$; a low proportion of non-English-speaking employees $(<30 \%)$; and a breakdown of $\leq 33 \%$ management/professional staff and $\geq 30 \%$ in blue- or pink-collar positions.

Eligible interested worksites were involved in a run-in phase that required a letter of intent to participate, an employee list for survey administration and a high baseline survey response rate. Worksites were recruited on a rolling basis, constituting four waves with thirty-two worksites from 2006 to 2008. Each wave contains six to ten worksites (three to five pairs) randomised to either intervention or control arms. The present study includes data from thirty-one worksites (one worksite was not randomised) at baseline only.

The study was conducted according to the guidelines laid down in the Declaration of Helsinki, and all procedures involving human subjects were approved by the Institutional Review Boards at the University of Washington and the Fred Hutchinson Cancer Research Center. Written informed consent was obtained from all participants.

\section{Baseline measurements}

Baseline surveys were solicited from all employees at participating worksites on an index date agreed with the company. Each site was given an additional 2 weeks to collect surveys from those who were not present at the proctored group meeting on the index date. If a site did not reach the $70 \%$ enrolment goal, two additional attempts were made to collect surveys from non-responders. The third and final attempts to collect survey data used a shorter version of the survey containing fifteen vital information questions. Additional measures (i.e. blood draw and pedometer assessment) were taken on a randomly selected subsample of about twenty individuals who had agreed on the survey to be re-contacted (or a minimum of $50 \%$ of survey respondents).

\section{Assessment of BMI}

BMI $\left(\mathrm{kg} / \mathrm{m}^{2}\right)$ was calculated using measured height and weight and was grouped according to standard categories: 'underweight'; 'normal'; 'overweight'; 'obese ${ }^{\text {(18) }}$. Measurements were conducted at the baseline survey administration for everyone who completed a survey, and at the date of a blood draw at the site for those missing these objective measures.

\section{Assessment of dietary behaviours}

Daily servings of fruits and vegetables were assessed via the survey, which provided pictorial representations of typical fruit and vegetable servings. Summary fruit and vegetable questions in a FFQ format ${ }^{(19)}$ assessed consumption of fruit, $100 \%$ fruit juice, other fruit juice, non-French-fried potatoes, non-potato vegetables and green salad. Fruit and vegetable consumption has consistently been shown to be associated with reduced $\mathrm{BMI}^{(20)}$. Several eating behaviours have been shown to be associated with the risk of obesity, including consumption of fast food ${ }^{(21)}$ and soft drinks ${ }^{(22)}$, television viewing $^{(22)}$, and eating while doing other activities ${ }^{(22)}$. The questionnaire included items on frequency of eating at fastfood restaurants, frequency of drinking sodas, and eating while doing another activity such as watching television.

\section{Assessment of physical activity}

Physical activity was quantified as free-time physical activity of at least $10 \mathrm{~min}$ and was assessed using a modified Godin Leisure-Time Exercise Questionnaire ${ }^{(23)}$, which used a cutpoint of $15 \mathrm{~min}$. The change, made in common with other centres in the Obesity Prevention in Worksites consortium, is

\footnotetext{
ised to either intervention or control arms. The present study
} 
consistent with the new recommendations ${ }^{(24)}$. Godin \& Shephard ${ }^{(25)}$ established its reliability (test-retest correlation coefficient ranging from 0.48 for light activity to 0.94 for strenuous activity) and validity in relation to maximal oxygen consumption. At least one additional study has confirmed reliability and validity ${ }^{(26)}$. Their published composite freetime exercise score was computed for each individual.

\section{Assessment of obesity-specific quality of life}

Obesity-specific QOL (HRQOL) was measured using the Obesity and Weight-Loss QOL (OWLQOL) Questionnaire ${ }^{(27,28)}$. The OWLQOL is a seventeen-item self-administered questionnaire that was developed cross-culturally to address shortcomings of pre-existing instruments that focus on functional status or behaviours associated with obesity. The theoretical foundation for this instrument is Maslow's needs hierarchy ${ }^{(29)}$. All items tap unobservable needs such as freedom from stigma and attainment of culturally appropriate goals. For example, respondents are asked the degree to which weight affects their energy with statements such as 'I feel frustrated that I have less energy because of my weight' and 'My weight prevents me from doing what I want to do'. Reliability and validity are well established and have exceeded recommended minimums within obese populations ${ }^{(28)}$. Guttman-Cronbach's $\alpha$ was 0.96 , indicating a high internal consistency, and factor analyses supported an overall score ${ }^{(28)}$. Although Cronbach's $\alpha$ of 0.96 is very high, it refers to the total scale and 6000 data points indicated a high $\alpha$ for each of many combinations of items, but the seventeen that were chosen reflected the best content ${ }^{(30)}$. Excluding several items reduced the $\alpha$ below 0.90, indicating reduced internal consistency ${ }^{(30)}$. The composite OWLQOL score was computed for each individual and used as the obesity-specific QOL outcome.

\section{Assessment of productivity}

Productivity was assessed using the eight-item short form of the Work Limitations Questionnaire (WLQ) ${ }^{(31)}$. The WLQ is a selfadministered questionnaire designed to measure the degree to which health problems may interfere with performing job functions ${ }^{(31)}$. For instance, respondents are asked 'In the past 2 weeks, how much of the time did your physical health or emotional problems make it difficult for you to do the following? (1) Get going easily at the beginning of the workday, (2) Handle the workload'. The reliability and validity of the WLQ are well established $^{(32)}$. The WLQ is considered to be a good measure of self-reported work productivity, as self-reported work limitations have been shown to be significantly $(P<0 \cdot 0001)$ associated with employee work productivity ${ }^{(32)}$. The overall WLQ Productivity Loss Score ${ }^{(33)}$ was computed, where a higher score indicates greater limitations in work productivity.

\section{Statistical methods}

Baseline demographic characteristics of the study subjects with no missing values for sex were computed. Associations between the log-transformed outcomes (OWLQOL scores and productivity loss scores) and the predictors were assessed. Predictors included log-transformed BMI, physical activity (Godin score), daily servings of fruits and vegetables, doing something while eating, fast-food restaurant meals per month and soft drink consumption. Multiplicative interaction terms were generated by creating a cross-product term between each predictor and sex, and tested for significance in a univariate model using a likelihood ratio test. Sex was found to modify the relationship between OWLQOL and all six predictors (each $P<0.001$ ), and between some predictors and WLQ productivity scores, so the OWLQOL and WLQ scores were analysed by sex. For both outcomes, adjusted geometric means and 95\% CI were obtained using linear mixed models for fixed age, race, education, and income effects and random worksite effects. The geometric mean is a common alternative way of presenting an average with an emphasis on central tendency, and is the appropriate choice when an outcome is log-transformed. It is similar to the arithmetic mean but is calculated by multiplying the numbers together (not summing, as in the arithmetic mean), and then dividing the resulting product by the $n$th root (where $n$ is the count of numbers in the set). Regression analyses were based on individuals with complete data on age, race, education, income, and the predictor and outcome of interest for each regression; thus, the number of individuals varied for each analysis. The outcomes were log-transformed to reduce skewness and back-transformed for ease of interpretation.

Next, OWLQOL scores and productivity loss scores were examined for a linear trend within each of the ordered categorical predictor variables described above, adjusting for age, race, education and income. Race was collapsed into five categories (non-Hispanic White, Hispanic, Asian, Black or African American, and Other) when used as an adjustment variable. Logarithmically transformed slope estimates and their corresponding standard errors were obtained, and then backtransformed by taking the exponential of the slopes and corresponding $95 \%$ CI. The exponential of the slope represented the relative change of the outcome when the predictor increased one unit. Finally, the effects of physical activity and obesogenic dietary behaviours were assessed when controlling for BMI, which was logarithmically transformed to reduce the effect of skewness.

All statistical tests were two-sided. Statistical analyses were conducted using Statistical Analysis Systems software (version 9.1; SAS Institute, Inc.).

\section{Results}

Results include all four randomisation waves of MOVE 'M (thirty-one worksites). The average worksite had twentyseven employees, similar to the non-randomised companies in the recruiting pool. The most common worksite size was fifteen to twenty-four employees for randomised companies and ten to fourteen for non-randomised companies. Randomised worksites reflected the Standard Industrial Classification code distribution of the underlying recruiting pool.

The original dataset of employees in randomised companies who completed the baseline questionnaire ( $n$ 753) was restricted to individuals with non-missing values for sex 
( $n 747,99 \cdot 2 \%$ of original dataset). A slightly greater proportion of males had a college degree, whereas a slightly greater proportion of females had a postgraduate or professional degree (Table 1). The majority of both female and male employees were non-Hispanic White (65.4 and 66.8\%, respectively), reflecting the general population composition in the Pacific Northwest. Approximately 61\% ( $n$ 459) of employees were either overweight $(25 \cdot 8 \%)$ or obese (35.6\%). The average BMI, servings of fruits and vegetables, free-time physical activity scores and WLQ scores were similar

Table 1. Baseline demographic characteristics of employees at Move and Moderate in Balance worksites (Number of participants and percentages; mean values and standard deviations)

\begin{tabular}{|c|c|c|c|c|}
\hline & \multicolumn{2}{|c|}{ Females ( $n$ 399) } & \multicolumn{2}{|c|}{ Males ( $n$ 348) } \\
\hline \multicolumn{5}{|l|}{ Age (years) } \\
\hline $25-39$ & 154 & 38.6 & 132 & 37.9 \\
\hline $40-54$ & 157 & 39.4 & 116 & 33.3 \\
\hline $55+$ & 58 & 14.5 & 67 & $19 \cdot 3$ \\
\hline Black or African American & 40 & $10 \cdot 0$ & 25 & $7 \cdot 2$ \\
\hline Asian & 37 & 9.3 & 36 & $10 \cdot 3$ \\
\hline Other† & 27 & $6 \cdot 8$ & 22 & $6 \cdot 3$ \\
\hline \multicolumn{5}{|l|}{ Education } \\
\hline Less than high school & 18 & 4.5 & 9 & $2 \cdot 6$ \\
\hline High school graduate or GED & 194 & $48 \cdot 6$ & 159 & $45 \cdot 7$ \\
\hline$\$ 25000-49000$ & 108 & $27 \cdot 1$ & 62 & $17 \cdot 8$ \\
\hline$\$ 50000-74999$ & 78 & $19 \cdot 6$ & 82 & 23.6 \\
\hline$\$ 75000-100000$ & 73 & $18 \cdot 3$ & 45 & $12 \cdot 9$ \\
\hline$>\$ 100000$ & 65 & $16 \cdot 3$ & 82 & 23.6 \\
\hline \multicolumn{5}{|l|}{ BMI $\left(\mathrm{kg} / \mathrm{m}^{2}\right)$} \\
\hline$<18.5$ ('underweight') & 5 & 1.3 & 0 & 0.0 \\
\hline 18.5-24.99 ('normal') & 122 & $30 \cdot 6$ & 62 & $17 \cdot 8$ \\
\hline 25-29.99 ('overweight') & 80 & $20 \cdot 1$ & 113 & 32.5 \\
\hline $30+$ ('obese') & 147 & 36.8 & 119 & 34.2 \\
\hline \multicolumn{5}{|l|}{ Daily servings of fruits/vegetables } \\
\hline$<1$ & 11 & $2 \cdot 8$ & 7 & $2 \cdot 0$ \\
\hline $1-2$ & 167 & 41.9 & 194 & $55 \cdot 8$ \\
\hline $3-4$ & 164 & $41 \cdot 1$ & 107 & 30.8 \\
\hline $5+$ & 56 & $14 \cdot 0$ & 37 & $10 \cdot 6$ \\
\hline \multicolumn{5}{|l|}{ Soft drink/soda (diet or regular) consumption } \\
\hline Seldom & 59 & $14 \cdot 8$ & 79 & $22 \cdot 7$ \\
\hline Sometimes & 161 & $40 \cdot 4$ & 137 & 39.4 \\
\hline \multirow[t]{2}{*}{ Most of the time/always } & 162 & $40 \cdot 6$ & 108 & $31 \cdot 0$ \\
\hline & Mean‡ & SD & Mean‡ & SD \\
\hline BMI $\left(\mathrm{kg} / \mathrm{m}^{2}\right)$ & $29 \cdot 7$ & $8 \cdot 1$ & $29 \cdot 8$ & $6 \cdot 0$ \\
\hline Servings of fruits/vegetables & 3.0 & 1.8 & $2 \cdot 6$ & $1 \cdot 8$ \\
\hline Fast-food meals per month & $5 \cdot 3$ & $6 \cdot 8$ & $6 \cdot 8$ & $7 \cdot 8$ \\
\hline Free-time physical activity score & $25 \cdot 1$ & $20 \cdot 6$ & $31 \cdot 3$ & 28.9 \\
\hline Obesity and Weight-Loss Quality-of-Life total score & 69.5 & 24.7 & $86 \cdot 0$ & $16 \cdot 3$ \\
\hline Work Limitations Productivity Loss Score & $6 \cdot 8$ & $6 \cdot 4$ & $6 \cdot 2$ & $6 \cdot 0$ \\
\hline
\end{tabular}

GED, General Equivalency Diploma.

* Percentages may not sum to $100 \%$ due to rounding or missing data.

†Other includes Native American or Alaskan Native, Hawaiian or Pacific Islander, or self-specified.

$\ddagger$ Arithmetic mean. 
Table 2. Mean Obesity and Weight-Loss Quality-of-Life (OWLQOL) scores by baseline reported dietary behaviours, physical activity and BMI (Mean values and $95 \%$ confidence intervals)

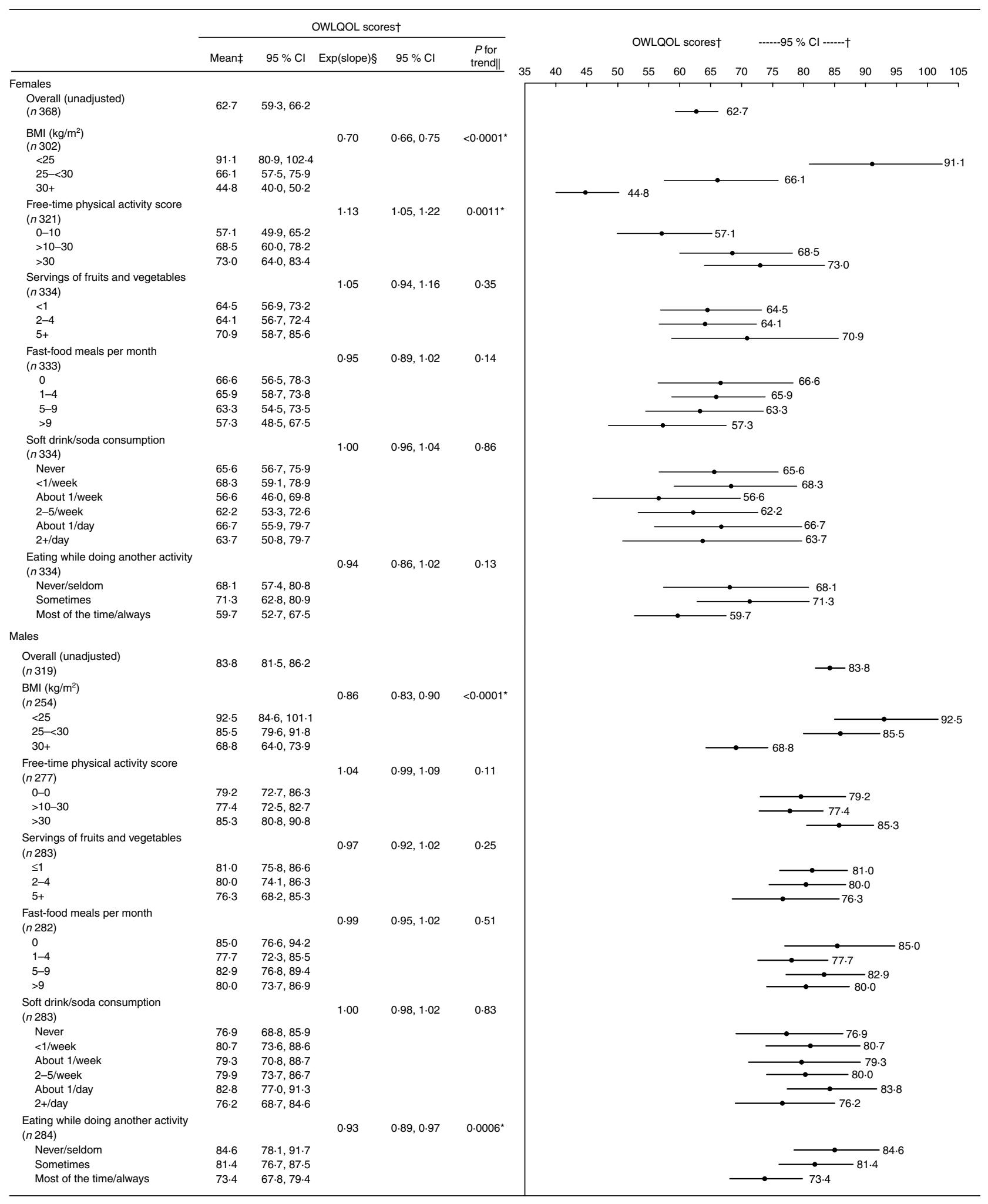

* Values were significantly different $(P<0.05)$

$\dagger$ Linear mixed model adjusted for age (continuous), race (collapsed into four categories), education and income.
$\ddagger$ Geometric mean.

$\S$ Exponential transform of slope estimate: slope coefficients were obtained from log-transformed data, but have been back-transformed for presentation.

॥ Trend tested with the Wald test. 
Table 3. Mean Work Limitations Questionnaire (WLQ) Productivity Loss Score by baseline reported dietary behaviours, physical activity and BMI (Mean values and $95 \%$ confidence intervals)

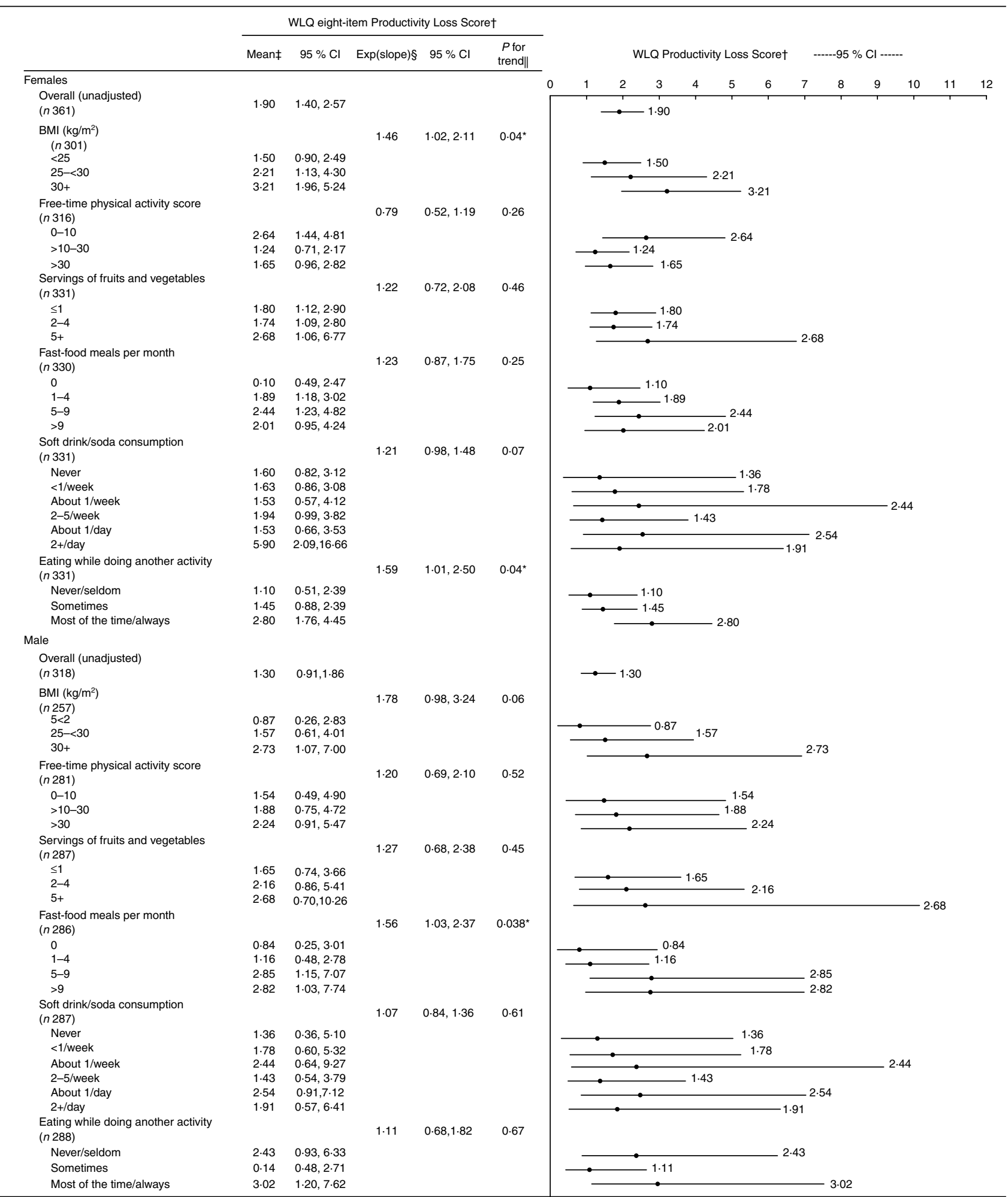

* Values are significantly different $(P<0 \cdot 05)$.

$\dagger$ Linear mixed model adjusted for sex, age (continuous), race (collapsed into 4 categories), education and income.

¥ Geometric mean.

$\S$ Exponential transform of slope estimate: slope coefficients were obtained from log-transformed data, but have been back-transformed for presentation here.

II Trend tested with the Wald test. 
between males and females; however, a higher proportion of men were overweight when compared with women. Males had a slightly higher average OWLQOL score (86.0) than females (69.5).

Examining OWLQOL as the outcome of interest, for all predictors, there were significant interactions with sex (each $P<0 \cdot 0001)$. Thus, all OWLQOL analyses were presented as sex-specific (Table 2). OWLQOL scores were negatively associated with BMI for both females $(P<0 \cdot 0001)$ and males $(P<0.0001)$. The negative impact of obesity on OWLQOL scores was more pronounced for women. An additional unit in BMI was associated with a 30\% lower (95\% CI 25, 34\%) geometric mean OWLQOL for women and a $14 \%$ lower (95\% CI 10, 17\%) geometric mean OWLQOL for men.

Physical activity was positively associated with OWLQOL only in women $(P=0 \cdot 0011)$. An additional unit in free-time physical activity score was associated with a $13 \%$ positive difference (95\% CI 5, 22\%) in geometric mean OWLQOL scores. However, this effect became non-significant when controlling for BMI. Servings of fruits and vegetables, soda consumption and fast food consumption were not significantly associated with OWLQOL scores for males or females. Eating while doing other activities was significantly associated with OWLQOL scores in men only ( $P=0.0006)$. The geometric mean OWLQOL score for men who ate more frequently while doing other activities was $7 \%$ lower (95\% CI 3, 11\%) than men who ate less frequently while doing other activities. This association remained significant after controlling for BMI but became attenuated (exp(slope) $=0.95,95 \%$ CI 0.92, 0.98, $P=0 \cdot 0028$ ). All other effects remained the same when controlling for BMI. All associations and effect sizes persisted after controlling for age, race, education and income.

Interactions were again present when examining the WLQ score as the outcome, so these analyses were also presented as sex-specific (Table 3). BMI was positively associated with WLQ scores only in women $(P=0 \cdot 04)$. A one-category difference in BMI was associated with a 1.46 -fold (95\% CI 1.02 , 2.11) positive difference in geometric mean WLQ scores. The association between BMI and WLQ scores was nonsignificant in men $(P=0.06)$. Eating while doing another activity was positively associated with WLQ scores only in women $(P=0 \cdot 04)$. A one-category difference was associated with a 1.59 -fold $(95 \%$ CI $1.01,2.50)$ positive difference in geometric mean WLQ scores; however, this effect diminished when controlling for BMI. Fast food consumption was significantly positively related to WLQ scores in men $(P=0.038)$. A one-category difference was associated with a 1.56-fold (95\% CI 1.03, 2.37) positive difference in geometric mean WLQ scores. This association remained significant when controlling for BMI. None of the remaining predictors was associated with productivity loss scores for males or females. All other effects remained the same when controlling for BMI, and all associations and effect sizes persisted after controlling for age, race, education and income.

\section{Discussion}

Results from the present study suggest that BMI is inversely associated with self-reported, obesity-specific HRQOL and work productivity. Certain obesogenic behaviours were also related to obesity-specific HRQOL and productivity. For women, free-time physical activity was associated with higher obesity-specific QOL, and eating while doing another activity was associated with lower productivity, but these associations appeared to operate through BMI. For men, eating while doing another activity was inversely associated with obesity-specific QOL and fast-food meals were inversely associated with productivity; however, unlike in women, these effects did not appear to operate through BMI.

The suggestion of a sex difference appears to be supported by the research in this area. As was expected from the literature $^{(28,34-36)}$, women in the present study were more likely to report lower obesity-specific QOL. In addition, the results presented agree with previous studies that found the impact of obesity on HRQOL to be more severe for women than for men ${ }^{(34,37)}$. Similarly, prior results have found an effect of physical activity on HRQOL for both men and women $^{(14,15)}$, and within women alone ${ }^{(38)}$. The findings also complement a study by Muennig et al. ${ }^{(39)}$ who found the desire to lose weight was a stronger predictor of unhealthy days for women than for men, concluding that negative body image contributes to the morbidity of obesity, particularly for women. Indeed, one potential explanation for this sex difference is body image. The social acceptability of body size can vary between women and men; at least one study has shown that overweight men are significantly more likely to report that their body size was socially acceptable than overweight women ${ }^{(40)}$. Women also tend to be more heavily affected by body image psychologically than men, associating body dissatisfaction with self-esteem ${ }^{(41)}$, and by selecting body images thinner than their own as being more desirable to the opposite $\operatorname{sex}^{(42)}$. Thus, it is possible that the positive effects of healthier dietary and physical activity behaviours on QOL and productivity are conditional on body size perceptions only among women.

The evidence presented, combined with current economic evidence, may help to convince management to invest in the health of their employees. In particular, the findings may have important implications for companies and organisations, which may want to consider offering healthier choices and lunch breaks for employees in order to maintain or increase productivity. It is especially important for the MOVE 'M study, as this could provide incentive for worksites to join the study if they believe that decreasing employees' BMI as a result of the intervention's effort may increase productivity.

The present study has several limitations. All variables, with the exception of BMI, were assessed using self-report survey data. However, it is important to note that QOL can only be measured by reports by the individual and perceived productivity may be an equally or more important measure compared with productivity measures such as absenteeism, lost wages and replacement staff from the employer. Due to the cross-sectional nature of these data, one cannot draw 
conclusions concerning causality. Thus, it is unclear whether BMI and physical activity may influence obesity-specific QOL, or if this relationship is reversed. Weight-related QOL could well be a predictor of weight-related behaviours. Due to the small number of underweight individuals, the data could not be used to verify previous research ${ }^{(43,44)}$, suggesting that individuals at either end of the spectrum, underweight or overweight/obese, tend to have compromised self-reported HRQOL. Nonetheless, whereas current studies have focused on general HRQOL, the present study confirms a similar negative impact of obesity on obesity-specific HRQOL. Finally, although the most common worksite size of randomised companies was slightly larger than non-randomised companies, this is unlikely to affect the generalisability of these results.

The study has several noteworthy strengths. The large sample size allows us to draw strong conclusions. In addition, height and weight were directly measured for all employees, resulting in BMI calculations that are more accurate than those obtained from self-reported height and weight. Using linear mixed models, worksite-level effects were controlled for in order to examine individual-level associations. Finally, the study design will allow us to evaluate the effect of the intervention, particularly in relation to the measures described in the present study. The hypothesis is that the intervention will increase fruit and vegetable consumption and physical activity and decrease BMI, and that a related improvement in OWLQOL and productivity scores will be observed. Nonetheless, this determination cannot be made based on cross-sectional data, and evaluation of these outcomes at 2-year follow-up may shed more light on this potential mediating mechanism.

These results suggest that obesity-specific HRQOL and productivity may be particularly impaired in obese men and women, with the impact being more pronounced in women. Physical activity and obesogenic behaviours may operate through BMI to affect obesity-specific QOL and productivity in women, but obesogenic behaviours appear to affect obesity-specific QOL and productivity independent of BMI in men. The implication of sex differences in the effects of obesity, physical activity and obesogenic behaviours on obesityspecific HRQOL and productivity is important and should be further researched. Such information may prove useful in addressing the mental health and overall well-being of obese women and men.

\section{Acknowledgements}

The authors wish to acknowledge the employees at the worksites who took the time to complete the baseline survey and physical measurements for the MOVE 'M study. Their voluntary participation made this investigation possible. The present study was supported by funding from the US National Cancer Institute as part of U54 CA116847. All authors contributed to this manuscript. S. W. C. conceived the study and wrote the majority of the manuscript. S. A. A. B. is the principal investigator of the MOVE ' $\mathrm{M}$ project, wrote the protocol, guided the assessment development, and monitored the research process for this paper from its inception by providing methodological and statistical guidance and participating in manuscript revisions. J. A. H. assisted in writing the Methods section, particularly concerning the recruitment of worksites. A. M. edited the manuscript and acted as the principal investigator of the collection of studies of which this study was a part. L. X. conducted all analyses and assisted in the write-up of the Statistical methods section. C. Y. W. contributed to the statistical methods section and aided in analyses. D. L. P. provided guidance on patient-reported outcomes and assisted in writing the sections on the measurement of obesity-related QOL (the OWLQOL) and the discussion and interpretation of the results. The authors declare that they have no competing interests.

\section{References}

1. US Department of Health and Human Services (2010) Overweight and obesity: introduction. http://www.cdc.gov/ obesity/data/trends.html (accessed 22 February 2010).

2. US Department of Health and Human Services (2010) Overweight and obesity: health consequences. http://www.cdc. gov/obesity/causes/health.html (accessed 22 February 2010).

3. US Department of Health and Human Services (2001) Office of Disease Prevention and Health Promotion. Healthy People 2010. Washington, DC. http://www.healthypeople.gov/ 2010/ (accessed 22 February 2010).

4. Karlsson J, Taft C, Ryden A, et al. (2007) Ten-year trends in health-related quality of life after surgical and conventional treatment for severe obesity: the SOS intervention study. Int J Obes (Lond) 31, 1248-1261.

5. Patrick DL \& Erickson P (1993) Health Status and Health Policy: Quality of Life in Health Care Evaluation and Resource Allocation. New York: Oxford University Press.

6. Fontaine KR, Cheskin LJ \& Barofsky I (1996) Health-related quality of life in obese persons seeking treatment. $J$ Fam Pract 43, 265-270.

7. Kolotkin RL, Crosby RD \& Williams GR (2002) Health-related quality of life varies among obese subgroups. Obes Res $\mathbf{1 0}$, 748-756

8. Wolf AM \& Colditz GA (1998) Current estimates of the economic cost of obesity in the United States. Obes Res 6, 97-106.

9. Finkelstein EA, Dibonaventura MD, Burgess SM, et al. (2010) The costs of obesity in the workplace. J Occup Environ Med 52, 971-976.

10. Sullivan PW, Ghushchyan V \& Ben-Joseph RH (2008) The effect of obesity and cardiometabolic risk factors on expenditures and productivity in the United States. Obesity 16, 2155-2162.

11. Bungum T, Satterwhite M, Jackson AW, et al. (2003) The relationship of body mass index, medical costs, and job absenteeism. Am J Health Behav 27, 456-462.

12. Plaisted CS, Lin PH, Ard JD, et al. (1999) The effects of dietary patterns on quality of life: a substudy of the Dietary Approaches to Stop Hypertension trial. J Am Diet Assoc 99, Suppl. 8, S84-S89.

13. Vuillemin A, Boini S, Bertrais S, et al. (2005) Leisure time physical activity and health-related quality of life. Prev Med 41, 562-569.

14. Brown DW, Balluz LS, Heath GW, et al. (2003) Associations between recommended levels of physical activity and health-related quality of life. Findings from the 2001 Behavioral Risk Factor Surveillance System (BRFSS) survey. Prev Med 37, 520-528.

15. Brown DW, Brown DR, Heath GW, et al. (2004) Associations between physical activity dose and health-related quality of life. Med Sci Sports Exerc 36, 890-896. 
16. Beresford SA, Thompson B, Feng Z, et al. (2001) Seattle 5 a Day worksite program to increase fruit and vegetable consumption. Prev Med 32, 230-238.

17. US Census Bureau (1997) SIC matched to 1997 NAICS major groups (2-Digit). http://www.census.gov/epcd/naics/nsic2ndx. htm (accessed 4 July 2008).

18. US Centers for Diseases Control and Prevention (2008) How is BMI calculated and interpreted? http://www.cdc.gov/ healthyweight/assessing/bmi/adult_bmi/index.html\#Interpreted (accessed 28 July 2008).

19. Thompson FE \& Byers T (1994) Dietary assessment resource manual. J Nutr 124, Suppl. 11, 2245S-2317S.

20. Lin BH \& Morrison RM (2002) Higher fruit consumption linked with lower body mass index. Food Rev 25, 28-32.

21. French SA, Harnack L \& Jeffery RW (2000) Fast food restaurant use among women in the Pound of Prevention study: dietary, behavioral and demographic correlates. Int $J$ Obes Relat Metab Disord. 24, 1353-1359.

22. Liebman M, Pelican S, Moore SA, et al. (2003) Dietary intake, eating behavior, and physical activity-related determinants of high body mass index in rural communities in Wyoming, Montana, and Idaho. Int J Obes Relat Metab Disord. 27, 684-692.

23. Godin G \& Shephard RJ (1997) Godin leisure-time exercise questionnaire. Med Sci Sports Exer 29, S36-S38.

24. US Department of Agriculture (2010) How much physical activity is needed? http://www.choosemyplate.gov/food groups/physicalactivity_amount.html (accessed 7 November 2010).

25. Godin G \& Shephard RJ (1985) A simple method to assess exercise behavior in the community. Can J Appl Sport Sci 10, 141-146.

26. Jacobs DR Jr, Ainsworth BE, Hartman TJ, et al. (1993) A simultaneous evaluation of 10 commonly used physical activity questionnaires. Med Sci Sports Exerc 25, 81-91.

27. Niero M, Martin M, Finger T, et al. (2002) A new approach to multicultural item generation in the development of two obesity-specific measures: the Obesity and Weight Loss Quality of Life (OWLQOL) questionnaire and the WeightRelated Symptom Measure (WRSM). Clin Ther 24, 690-700.

28. Patrick DL, Bushnell DM \& Rothman M (2004) Performance of two self-report measures for evaluating obesity and weight loss. Obes Res 12, 48-57.

29. Maslow AH (1943) A theory of human motivation. Psychol Rev 50, 370-396.
30. Patrick D (2010) Personal communication via e-mail. [S Whisnant, editor].

31. Lerner D, Amick BC 3rd, Rogers WH, et al. (2001) The work limitations questionnaire. Med Care 39, 72-85.

32. Lerner D, Amick BC 3rd, Lee JC, et al. (2003) Relationship of employee-reported work limitations to work productivity. Med Care 41, 649-659.

33. Lerner D, Rogers WH \& Chang H (2005) Technical report: Scoring the Work Limitations Questionnaire (WLQ) scales and the WLQ index for estimating work productivity loss. Unpublished report.

34. Kolotkin RL, Head S, Hamilton M, et al. (1995) Assessing impact of weight on quality of life. Obes Res 3, 49-56.

35. Katz DA, McHorney CA \& Atkinson RL (2000) Impact of obesity on health-related quality of life in patients with chronic illness. J Gen Intern Med 15, 789-796.

36. Kolotkin RL, Crosby RD, Kosloski KD, et al. (2001) Development of a brief measure to assess quality of life in obesity. Obes Res 9, 102-111.

37. Larsson U, Karlsson J \& Sullivan M (2002) Impact of overweight and obesity on health-related quality of life - a Swedish population study. Int J Obes Relat Metab Disord 26, 417-424

38. Brown WJ, Mishra G, Lee C, et al. (2000) Leisure time physical activity in Australian women: relationship with well being and symptoms. Res $Q$ Exerc Sport 71, 206-216.

39. Muennig P, Jia H, Lee R, et al. (2008) I think therefore I am: perceived ideal weight as a determinant of health. $A m J$ Public Health 98, 501-506.

40. Rand CS \& Resnick JL (2000) The "good enough" body size as judged by people of varying age and weight. Obes Res $\mathbf{8}$, 309-316.

41. Furnham A, Badmin N \& Sneade I (2002) Body image dissatisfaction: gender differences in eating attitudes, self-esteem, and reasons for exercise. I Psychol 136, 581-596.

42. Demarest J \& Allen R (2000) Body image: gender, ethnic, and age differences. J Soc Psychol 140, 465-472.

43. Ford ES, Moriarty DG, Zack MM, et al. (2001) Self-reported body mass index and health-related quality of life: findings from the Behavioral Risk Factor Surveillance System. Obes Res 9, 21-31.

44. Jia H \& Lubetkin EI (2005) The impact of obesity on healthrelated quality-of-life in the general adult US population. J Public Health (Oxf) 27, 156-164. 